\title{
Different patterns of amygdala priming differentially affect dentate gyrus plasticity and corticosterone, but not CA1 plasticity
}

\author{
Rose-Marie Vouimba ${ }^{1}$ and Gal Richter-Levin ${ }^{2,3,4 *}$ \\ ${ }^{1}$ CNRS, Unité Mixte de Recherche 5287, Institut de Neurosciences Cognitives et Intégratives d'Aquitaine, Université de Bordeaux, Talence, France \\ 2 Department of Psychology, University of Haifa, Mount Carmel, Haifa, Israel \\ ${ }^{3}$ The Sagol Department of Neurobiology, University of Haifa, Mount Carmel, Haifa, Israel \\ ${ }^{4}$ Institute for the Study of Affective Neuroscience, University of Haifa, Mount Carmel, Haifa, Israel
}

\section{Edited by:}

Donald A. Wilson, New York

University School of Medicine, USA

Reviewed by:

Kei Cho, University of Bristol, UK

Jason S. Snyder, University of

British Columbia, Canada

*Correspondence:

Gal Richter-Levin, Brain and Behavior Laboratory, Department of

Psychology, University of Haifa, Rabin Building, Aba Chushi Rd., Mt. Carmel, Haifa 31905, Israel. e-mail:galrichterlevin@gmail.com
Stress-induced activation of the amygdala is involved in the modulation of memory processes in the hippocampus. However, stress effects on amygdala and memory remain complex. The activation of the basolateral amygdala (BLA) was found to modulate plasticity in other brain areas, including the hippocampus. We previously demonstrated a differential effect of BLA priming on long-term potentiation (LTP) in the CA1 and the dentate gyrus (DG). While BLA priming suppressed LTP in CA1, it was found to enhance it in the DG. However, since the amygdala itself is amenable to experience-induced plasticity it is thus conceivable that when activity within the amygdala is modified this will have impact on the way the amygdala modulates activity and plasticity in other brain areas. In the current study, we examined the effects of different patterns of BLA activation on the modulation of LTP in the DG and CA1, as well as on serum corticosterone (CORT). In CA1, BLA-priming impaired LTP induction as was reported before. In contrast, in the DG, varying BLA stimulation intensity and frequency resulted in differential effects on LTP, ranging from no effect to strong impairment or enhancement. Varying BLA stimulation patterns resulted in also differential alterations in Serum CORT, leading to higher CORT levels being positively correlated with LTP magnitude in DG but not in CA1. The results support the notion of a differential role for the DG in aspects of memory, and add to this view the possibility that DG-associated aspects of memory will be enhanced under more emotional or stressful conditions. It is interesting to think of BLA patterns of activation and the differential levels of circulating CORT as two arms of the emotional and stress response that attempt to synchronize brain activity to best meet the challenge. It is foreseeable to think of abnormal such synchronization under extreme conditions, which would lead to the development of maladaptive behavior.

Keywords: hippocampus, dentate gyrus, BLA, LTP, corticosterone, stress

\section{INTRODUCTION}

Stress-induced alterations in learning and memory processes are involved in the pathophysiology of stress-related disorders. Yet, stress effects on learning and memory are not only deleterious, but range from impairment to no effect to facilitation. The various effects of stress on memory functions may depend on stressors characteristics and on stress-induced release of stress hormones and neuromodulators (Shors, 2001; Baldi and Bucherelli, 2005; Joëls and Baram, 2009). Electrophysiological approaches revealed that long-term potentiation (LTP), a synaptic model of memory (Bliss and Collingridge, 1993), is also differentially altered by various stressors (Foy et al., 1987; Mesches et al., 1999; Kavushansky et al., 2006). The complexity of stress effects on hippocampal-dependent memory processes emerges also from its differential impact on hippocampal sub-regions. Thus, while stress and stress hormones have consistently been reported to impair LTP in CA1 (Pavlides et al., 1996; Xu et al.,
1998; Vouimba et al., 2006), the outcome on dentate gyrus (DG) LTP is more diversified (Bramham et al., 1998; Gerges et al., 2001; Yamada et al., 2003). For instance, discriminatory avoidance learning, which increases arousal, selectively inhibited LTP in CA1 but enhanced LTP in DG (Izaki and Arita, 1996). Predator stress enhanced LTP in DG for 5-7 days (Dringenberg et al., 2008), but impaired CA1 LTP (Vouimba et al., 2006). Moreover, increased release of CORT impaired CA1 LTP (Diamond et al., 1992; Pavlides et al., 1993), but had no effect on LTP in DG (Bramham et al., 1998).

The amygdala, an essential component of the neural network involved in emotional memory and stress-related behaviors, is a key mediator of stress effects on hippocampal-dependent memory processes (Roozendaal et al., 2003, 2004). Thus, while lesions or pharmacological suppression of the basolateral amygdala nucleus (BLA) were reported to impair DG LTP (Abe 2001), Kim et al. (2001) reported normal CA1 LTP in BLA lesioned 
stressed animals. Similarly, while BLA stimulation augments LTP in DG (Akirav and Richter-Levin, 1999), we later reported that BLA priming impairs CA1 LTP (Vouimba and Richter-Levin, 2005; Vouimba et al., 2007), further validating the claim that amygdalar modulation of hippocampal plasticity differs among its sub-fields.

Thus, an intact and active amygdala seems to be necessary for stress-induced alteration of hippocampal processes (Chauveau et al., 2008). However, although stress has been demonstrated to readily activate the amygdala, its effects on amygdala activity are rather complex. For instance, an acute platform stress enhanced BLA activity and LTP (up to 7 days), while repeated stress exposure inhibited BLA LTP (Vouimba et al., 2004). Thus, different stressors could activate the amygdala in ways that could be accountable for the differential effects of stress on memory processes (Tsoory et al., 2008). Furthermore, the amygdala itself is amenable to experienceinduced plasticity. It is thus conceivable that when activity within the amygdala is modified this will have impact on the way the amygdala modulates activity and plasticity in other brain areas.

In the present study, we further addressed the role of the BLA on CA1 and DG LTP by testing the impact of various patterns of BLA priming on hippocampal plasticity. In addition, since it has been indicated that amygdala activation may lead to the activation of the hypothalamic-pituitary-adrenal (HPA) axis (Goldstein et al., 1996; Feldman and Weidenfeld, 1998) we have also measures serum corticosterone (CORT) following the different patterns of BLA activation. We report that various patterns of BLA activation mainly inhibited LTP in CA1 but differentially modulated LTP in DG. In addition, various patterns of BLA activation enhanced CORT to different levels such that CORT levels positively correlated with LTP magnitude in DG but not in CA1.

\section{MATERIALS AND METHODS SUBJECTS}

Experiments were performed on male Sprague-Dawley rats (200-250 g), housed in Plexiglas cages and maintained on a freefeeding regimen with a 12:12 h light/dark schedule. Experiments were performed during the light phase of the cycle at least 1 week after their arrival from the supplier (Harlan Laboratories, Jerusalem, Israel). The experiments were performed in accordance with University of Haifa Ethics and Regulations and complied with the National Institute of Health guide for Care and Use of laboratory animals (NIH publication number 8023).

\section{SURGERY}

Rats were anesthetized with Equitisin $(42.5 \mathrm{mg} / \mathrm{ml}$ chloral hydrate, $21 \mathrm{mg} / \mathrm{ml}$ magnesium sulfate, and $9.7 \mathrm{mg} / \mathrm{ml}$ pentobarbital sodium; $0.4 \mathrm{ml} / 100 \mathrm{~g} \mathrm{ip}$ ) and mounted onto a stereotaxic frame (Stoelting, Wood Dale, IL, USA) and the head position was adjusted to place bregma and lambda in the same horizontal plane. The scalp was incised and retracted and small burr holes were drilled in the skull for the placement of stimulating and recording electrodes. Body temperature was monitored and maintained at $37^{\circ} \mathrm{C} \pm 0.5$ by a regulated heating pad during the course of the experiments.

\section{EVOKED FIELD POTENTIALS IN CA1 AND DG}

A recording glass electrode (tip diameter, 2-5 $\mu \mathrm{m}$; filled with $2 \mathrm{M}$ $\mathrm{NaCl}$ ) was stereotaxically positioned in the CA1 [4.2 $\mathrm{mm}$ posterior to bregma (AP), $2.5 \mathrm{~mm}$ from midline (ML), and $\sim 2 \mathrm{~mm}$ dorsoventral (DV) or in the DG $(4.2 \mathrm{~mm} \mathrm{AP,} 2.5 \mathrm{~mm} \mathrm{ML}$, and $\sim 3.7 \mathrm{~mm} \mathrm{DV})]$. Bipolar concentric stimulating electrodes (125 $\mu \mathrm{m}$; Kopf, Tujunga, CA, USA) were inserted in the ipsilateral BLA ( $3 \mathrm{~mm} \mathrm{AP,} 5.2 \mathrm{~mm} \mathrm{ML}$, and $7.6 \mathrm{~mm} \mathrm{DV}$ ) and either in the controlateral ventral hippocampal commissure (vHC: $2 \mathrm{~mm}$ AP, $1.5 \mathrm{~mm} \mathrm{ML}$, and $\sim 3 \mathrm{~mm} \mathrm{DV}$ ) for activating field potentials in the CA1 or in the ipsilateral perforant pathway (PP: $8 \mathrm{~mm} \mathrm{AP}$, $4 \mathrm{~mm} \mathrm{ML}$, and $\sim 3 \mathrm{~mm} \mathrm{DV}$ ) for activating field potentials in DG. The DV location of the recording and stimulating electrodes was adjusted to maximize the amplitude of evoked field potentials (EPs). A reference electrode consisting of a $100 \mu \mathrm{m}$ coated wire was affixed to the skull in the area overlapping the nasal sinus.

\section{STIMULATING AND RECORDING PROCEDURES}

CA1 and DG field potentials evoked by single pulses delivered to vHC or PP, respectively ( $0.1 \mathrm{~ms}$ rectangular monophasic pulses) were amplified $(\times 1000)$ by a A-M systems amplifier, displayed on an oscilloscope, digitized at $10 \mathrm{kHz}$ (CED) and stored to disk for off-line analysis (Signal-2 software). Baseline responses were established by means of stimulation intensity (50-200 $\mu \mathrm{A})$ sufficient to elicit a response representing $\sim 30 \%$ of the maximal amplitude of the EPs.

LTP was assessed by measuring the increase in the population spike amplitude (PS) of the EPs for both DG and CA1.

\section{PROTOCOLS}

Baseline recording of EPs in CA1 or DG was conducted for $30 \mathrm{~min}$ (one pulse every $30 \mathrm{~s}$ ). In the Control group baseline recording in CA1/DG was followed by application of a theta-like high frequency stimulation (TS): one set of 5 trains; each train consisted of 5 pulses at $100 \mathrm{~Hz}$; inter-train interval was $200 \mathrm{~ms}$ to the $\mathrm{vHC}$ or PP for LTP induction in CA1 or DG, respectively. After TS, responses to test pulse stimuli were recorded every $20 \mathrm{~s}$ for an additional $1 \mathrm{~h}$.

In the BLA priming groups, baseline recording was followed by various patterns of BLA stimulation $30 \mathrm{~s}$ prior TS. Responses to low frequency baseline pulses to the $\mathrm{vHC}$ or PP were then collected every $30 \mathrm{~s}$ for $1 \mathrm{~h}$. We used 8 experimental groups which differed by BLA stimulation frequency or strength.

\section{PATTERNS OF AMYGDALA STIMULATION}

Stimulation strength was modulated by varying the number of pulses in the protocol. Four Strength protocols were used: the first protocol consisted of 2 trains of five pulses at $100 \mathrm{~Hz}$; intertrain interval, $200 \mathrm{~ms} ; 1 \mathrm{~V}, 50 \mu \mathrm{s}$ pulse duration (BLA 2 trains). The second, third, and fourth protocols "BLA 10 trains," "BLA 15 trains," and "BLA 20 trains," respectively, consisted of constant frequency and pulse duration throughout the protocols, with a gradual increase of the number of trains to 10,15 , and 20, respectively.

BLA Stimulation frequency was modulated by varying train frequencies. Five protocols were used. Two high frequency stimulation (HFS) protocols: "BLA $100 \mathrm{~Hz}$ " consisting of 10 trains 
of five pulses at $100 \mathrm{~Hz}$; intertrain interval, $200 \mathrm{~ms} ; 1 \mathrm{~V}, 50 \mu \mathrm{s}$ pulse duration (identical to "BLA 10 trains"). "BLA $400 \mathrm{~Hz}$ " was identical to the previous group except that the pulses were delivered at $400 \mathrm{~Hz}$. Three low frequency stimulation (LFS) protocols: " $B L A 25 \mathrm{~Hz}$ " and "BLA $1 \mathrm{~Hz}$ " consisted of 10 trains of five pulses (50 pulses) at 25 and $1 \mathrm{~Hz}$, respectively. In addition, we used a classic LFS protocol (900 pulses at $1 \mathrm{~Hz}$ : “BLA $1 \mathrm{~Hz}, 900$ ”) known to readily elicit long-term depression as well as depotentiation in several brain regions (Wang et al., 1997; Vouimba et al., 2000).

\section{SERUM CORTICOSTERONE}

To assess the effect of BLA stimulations on serum CORT and establish a possible relation between CORT and hippocampal plasticity; in another sub-set of animals, stimulating and recording electrodes were positioned in the BLA for stimulation and in vHC-CA1 or PP-DG for LTP induction, as described above. In control animals, the stimulating electrode was lowered in the amygdala but no stimulation was then administered. Change in synaptic plasticity in all groups was recorded for $10 \mathrm{~min}$, then 5 min later (15 min following amygdala activation in experimental groups), animals were decapitated, trunk blood collected into plastic tubes, and allowed to clot for $1 \mathrm{~h}$ before centrifugation (3000 rpm for $15 \mathrm{~min}$ at $4^{\circ} \mathrm{C}$ ). Serum was aliquoted into storage microfuge tubes (Eppendorf) and stored at $-80^{\circ} \mathrm{C}$ until assayed for CORT levels, using enzyme immunosorbent assay kit (Assay Designs, Ann Arbor, MI, USA) according to manufacturer's instructions. The sensitivity for the CORT assay was $\sim 1.3 \mathrm{ng} / \mathrm{ml}$, and the average inter- and intra-assay covariance (\%) was less than 10 and 5\%, respectively. CORT results are presented as $\mathrm{ng} / \mathrm{mL}$. All blood samples were obtained during the light cycle (between 8:30 and 10:30 a.m.) at the nadir of the diurnal CORT rhythm.

\section{HISTOLOGY}

After completion of the studies, animals were either transcardially perfused with physiological saline, followed by $10 \%$ buffered formalin (electrophysiological study) or brains were directly remove after blood collection (CORT study). Brains were then post-fixed in formalin-saccharose $30 \%$ solution for at least 3 days and subsequently frozen, cut coronally on a sliding microtome into $80 \mu \mathrm{m}$ sections. The sections were mounted on gelatin-coated slides, and stained with cresyl violet for microscopic examination of the placements of the electrode in the BLA.

\section{DATA ANALYSIS}

The amplitude of the PS of the EPs was expressed as the mean percentage $( \pm$ SEM $)$ of the individual basal values of animals for each group. Groups differences were analyzed by ANOVA and post-hoc Fisher tests. A simple regression analysis was used to determine correlation between LTP magnitude and CORT. All statistical analyses were carried out with Statview.

\section{RESULTS HISTOLOGY}

Histological analysis showed that the stimulating electrode was mainly located in the BLA (Figure 1). Only rats with correct electrode positioning in the BLA were included for further analysis.

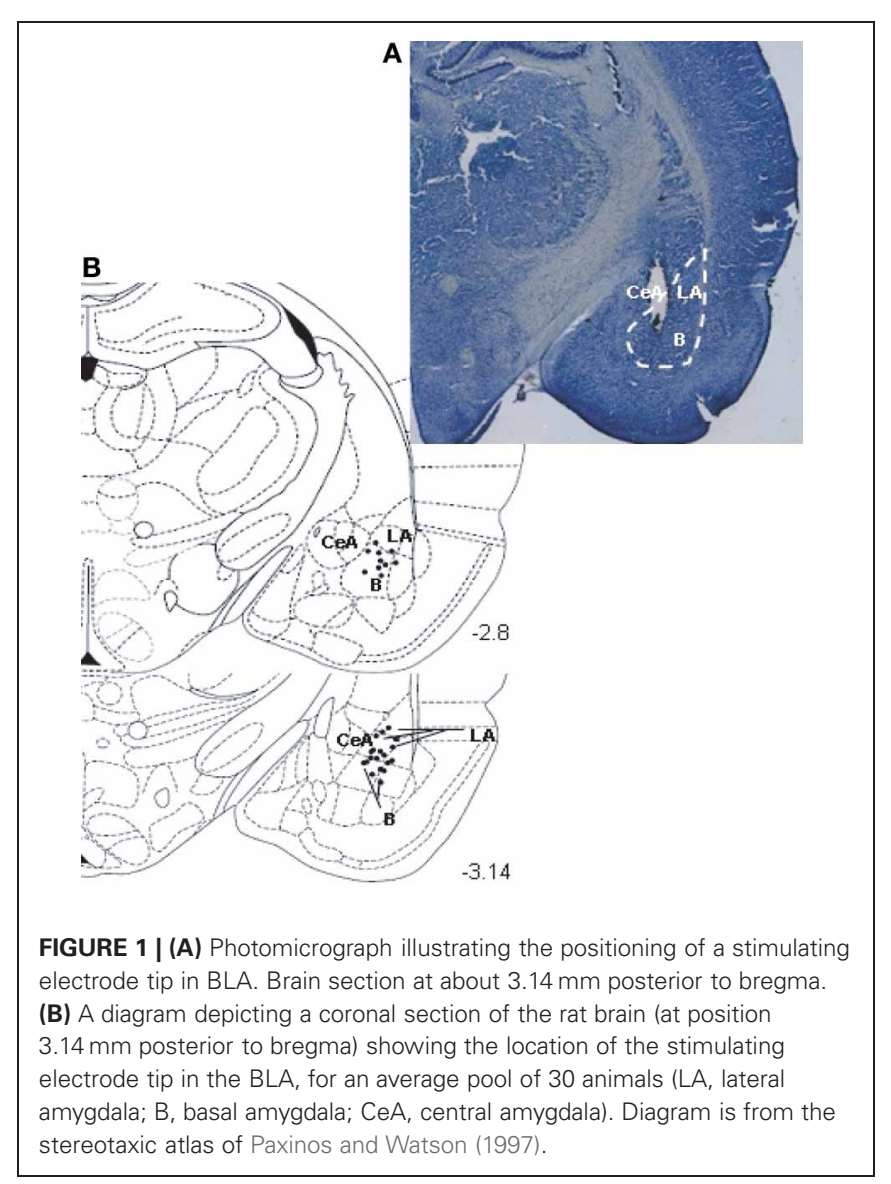

The indicated numbers of rats per groups are the final group sizes after histological control of electrode location.

\section{EFFECTS OF FREQUENCY MODULATION OF BLA ACTIVATION ON LTP IN CA1 AND DG}

In the attempt to understand the effects of frequency modulation of amygdala activation on LTP in CA1 and DG, we used low and high frequency BLA stimulation patterns (LFS and HFS- Figure 2). Priming the BLA with HFS, 100 or $400 \mathrm{~Hz}$, impaired LTP in vHC-CA1 pathway (\% changes for the last 10 min: CONT: $357.1 \pm 21.6 \% ; B L A-100 \mathrm{~Hz}: 227.4 \pm 26.6 \%$; $B L A-400 \mathrm{~Hz}: 209.5 \pm 35.4 \%$ ). ANOVAs performed on these data revealed a significant difference between control and BLA priming groups $\left[F_{(2,20)}=8.1 ; p<0.01\right]$. No significant difference was observed between the $B L A-100 \mathrm{~Hz}$ and $B L A-400 \mathrm{~Hz}$ groups $(p=0.45)$.

When BLA was primed using LFS, $25 \mathrm{~Hz}$ and classic LFS pattern (900 pulses at $1 \mathrm{~Hz}$ ) impaired LTP in the CA1, while $1 \mathrm{~Hz}$ (50 pulses) had no effect on LTP (\% changes for the last $10 \mathrm{~min}: B L A-25 \mathrm{~Hz}: 178.6 \pm 14.5 \%$; BLA-1 Hz: $353.8 \pm$ $16.36 \%)$. ANOVAs revealed a significant difference between groups $\left[F_{(3,21)}=19.7 ; p<0.0001\right]$. Post-hoc Fisher tests showed a significant difference between $B L A-25 \mathrm{~Hz}$ and both control and $B L A-1 \mathrm{~Hz}(p<0.0001)$, as well as between $B L A-1 \mathrm{~Hz}, 900$ and both control and $B L A-1 \mathrm{~Hz}$ groups $(p<0.0001)$ without any difference between control and $B L A-1 \mathrm{~Hz}(p=0.65)$. 


\section{CA1}

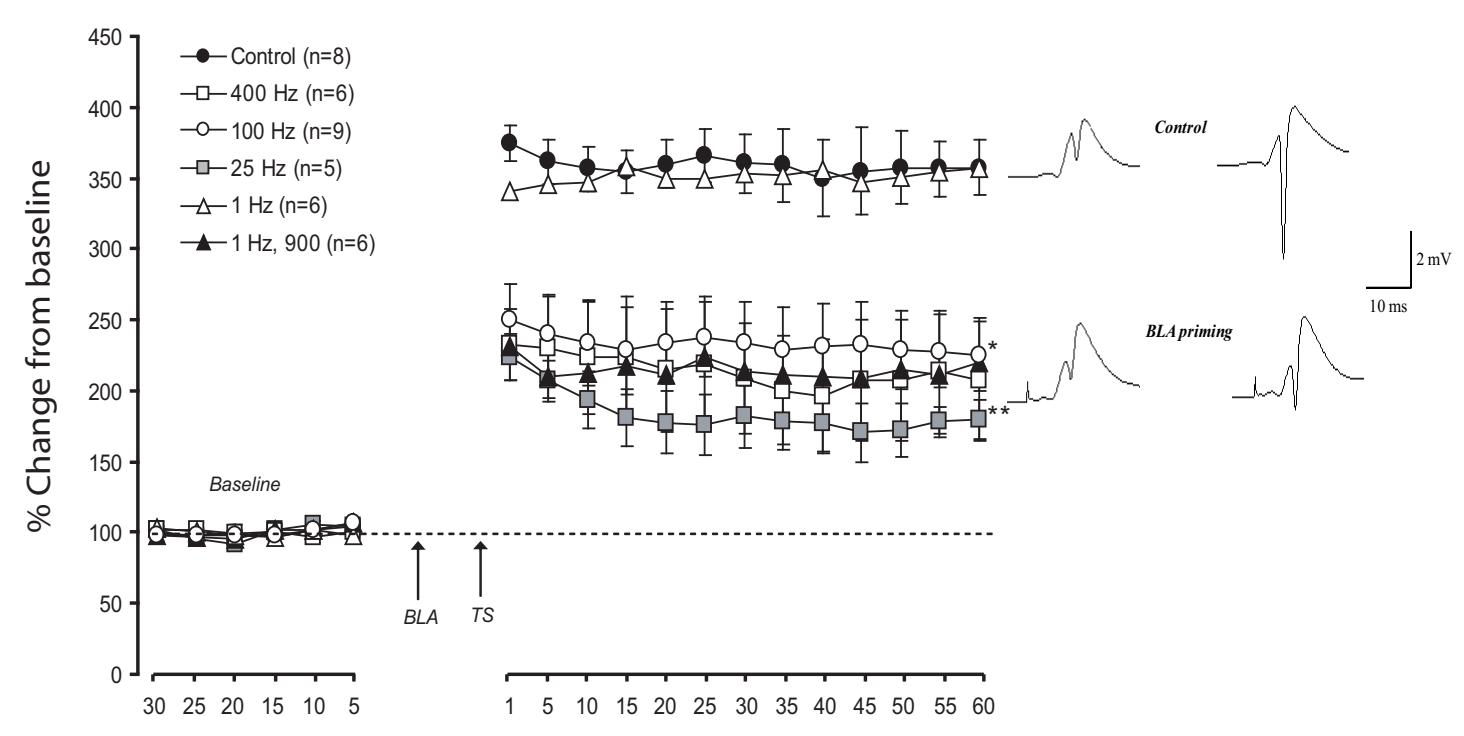

Time ( $\min )$

DG

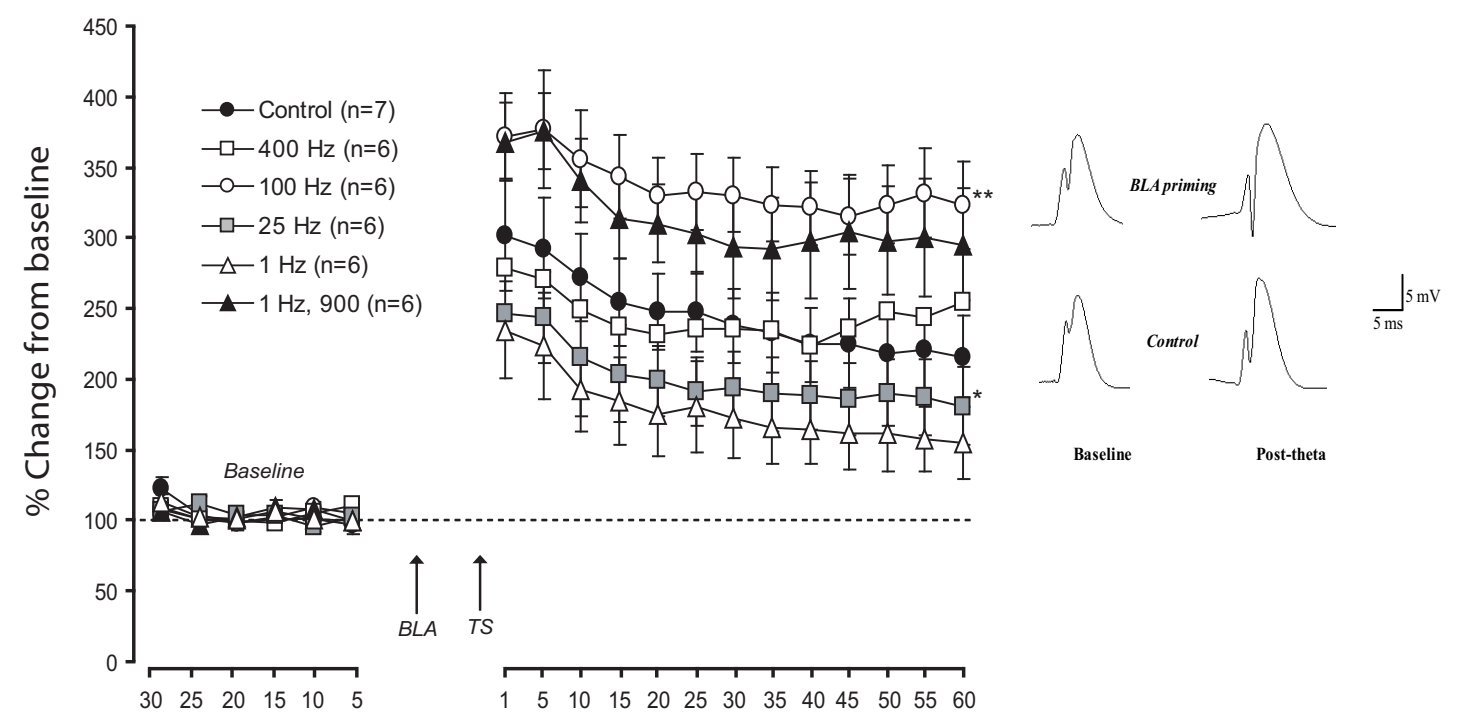

Time ( $\min )$

FIGURE 2 | Effects of frequency modulation of BLA activation on LTP in CA1 and DG: Mean ( \pm SEM) percentage of baseline. The majority of patterns of BLA activation used here impaired LTP in CA1. In DG, the different patterns induced changes varying from enhancement to strong impairment.
The pattern less favorable for DG $(1 \mathrm{~Hz})$ was the only one which did not interfere with CA1 plasticity. Only one pattern $(25 \mathrm{~Hz})$ induced similar deficit in DG and CA1 LTP. Post-hoc Fischer test: ${ }^{*} p<0.05$ and ${ }^{* *} p<0.001$ significantly different from control groups.
As previously reported (Vouimba and Richter-Levin, 2005; Vouimba et al., 2007) priming the BLA with $100 \mathrm{~Hz}$ HFS enhanced LTP in DG (\% changes for the last $10 \mathrm{~min}$ : CONT: $213.3 \pm 27.9 \%$; BLA-100 Hz: $320 \pm 30 \%)$. However, a $400 \mathrm{~Hz}$ HFS did not affect LTP (\% changes for the last $10 \mathrm{~min}$ :
BLA-400 Hz: $243.2 \pm 36.2 \%)$. ANOVA revealed a significant difference between groups $\left[F_{(2,16)}=3.9 ; p<0.05\right]$. A post-hoc Fisher test revealed a significant difference between $100 \mathrm{~Hz}$ and both CONT and $400 \mathrm{~Hz}(p<0.0001)$ with no significant difference between CONT and $400 \mathrm{~Hz}(p=0.95)$. 
When the BLA was primed with LFS, both 1 and $25 \mathrm{~Hz}$ patterns impaired LTP in the DG compared to control (\% changes for the last $10 \mathrm{~min}: B L A-25 \mathrm{~Hz}: 181.2 \pm 27.3 \%$; BLA-1 Hz: $153.3 \pm$ $24.5 \%$ ). In addition, unlike in the vHC-CA1 pathway, 900 pulses at $1 \mathrm{~Hz}$ enhanced LTP in DG (\% changes for the last $10 \mathrm{~min}$ for BLA-1 Hz,900: $298 \pm 33 \%)$. Statistical analysis revealed a significant difference between groups $\left[F_{(3,20)}=4.5 ; p=0.014\right]$. Pairwise comparisons showed a significant LTP reduction in both $B L A-25 \mathrm{~Hz}$ and $B L A-1 \mathrm{~Hz}$ as compared with control $(p<0.01)$. LTP in the $B L A-1 \mathrm{~Hz}, 900$ pulses was significantly enhanced as compared to the control group $(p<0.001)$.

\section{MODULATION OF STRENGTH OF BLA ACTIVATION ON LTP IN CA1 AND DG}

Results are seen on Figure 3.

Varying the strength of BLA activation, we found consistent impairment of vHC-CA1 LTP with all patterns used. Interestingly, the strongest impairment was seen with the weakest pattern (BLA-2 trains) while lighter changes accompanied stronger patterns of stimulation (\% changes for the last 10 min: CONT: $357.1 \pm 21.6 \%$; BLA-2 trains: $222.3 \pm 37 \%$; BLA-10 trains: $227.4 \pm 26.6 \%$; BLA-15 trains: $252.8 \pm 39 \%$; BLA20 trains: $306.86 \pm 32 \%)$. ANOVA revealed a significant difference between groups $\left[F_{(4,33)}=4.97 ; p=0.003\right]$. A post-hoc Fisher test showed a significant difference between control and all BLA priming groups $(p<0.05)$. Significant pairwise comparisons revealed significant differences between the BLA priming groups $(p<0.05)$ except for 2 trains and 10 trains which were not significantly different from each other $(p=0.7)$.

Unlike the CA1, changes in LTP in the PP-DG pathway were non-linear ( $\%$ changes for the 10 last min: CONT: $213.3 \pm 27.9 \% ; \quad B L A-2$ trains: $201 \pm 22.6 \%$; BLA-10 trains: $320 \pm 30 \%$; BLA-15 trains: $156.5 \pm 31 \%$; BLA-20 trains: $132 \pm$ $10.4 \%)$. ANOVA revealed a significant difference between groups $\left[F_{(4,27)}=7.85 ; p<0.001\right]$. A post-hoc Fisher test showed a significant difference between control and all BLA priming groups $(p<0.05)$, except for the 2 trains group, which was not significantly different from control $(p=0.06)$. Significant pairwise comparisons revealed significant differences between BLA priming groups $(p<0.05)$ except for the 15 trains vs. 20 trains groups, which were not significantly different from each other $(p=0.8)$.

In summary, comparing the effects of different patterns of BLA stimulation we found a dichotomy between CA1 and DG. The less efficient pattern for CA1 was best for DG and vice versa [except for two stimulation parameters (25 Hz and 15 trains) which impaired LTP in both CA1 and DG].

\section{AMYGDALA STIMULATION INCREASED LEVELS OF SERUM CORTICOSTERONE}

Amygdala stimulation is known to enhance serum CORT (Dunn and Orr, 1984; Vouimba et al., 2007). Here we examined if different patterns of BLA activation could differentially influence CORT. We selected BLA stimulation parameters which yield the strongest effects on both CA1 and DG plasticity. Thus, we investigated the effects of $400,100,25,1 \mathrm{~Hz}$, and $1 \mathrm{~Hz}, 900$ on CORT.

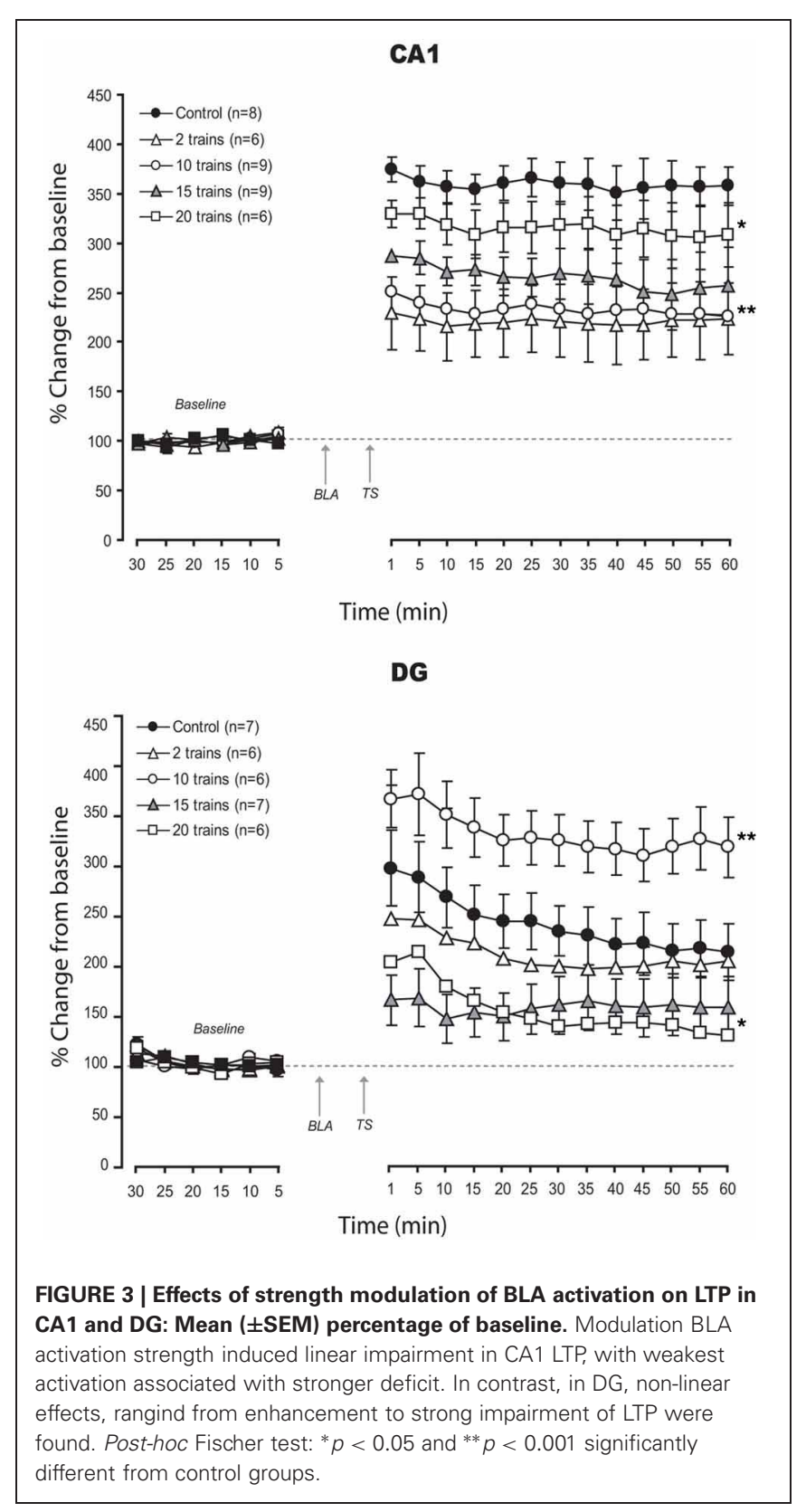

Serum samples analyses revealed a significant difference among groups $\left[F_{(5,28)}=24.35 ; P<0.0001\right.$; Figure 4]. The five BLA stimulation protocols used in this study significantly increased CORT as compared with control rats $(p<0.01)$. In addition, a post-hoc Fisher test showed that both $100 \mathrm{~Hz}$ and $1 \mathrm{~Hz}, 900$ groups exhibited significantly higher CORT level than the other stimulated groups $(p<0.001)$.

\section{CORRELATION BETWEEN SERUM CORTICOSTERONE AND HIPPOCAMPAL LTP}

Regression analyses revealed that the amplitude of DG LTP was positively correlated with CORT in BLA-stimulated animals $(r=0.75 ; p<0.0001)$. In contrast, no linear relation was observed between CA1 LTP and CORT ( $r=0.004 ; p=0.98)$. 


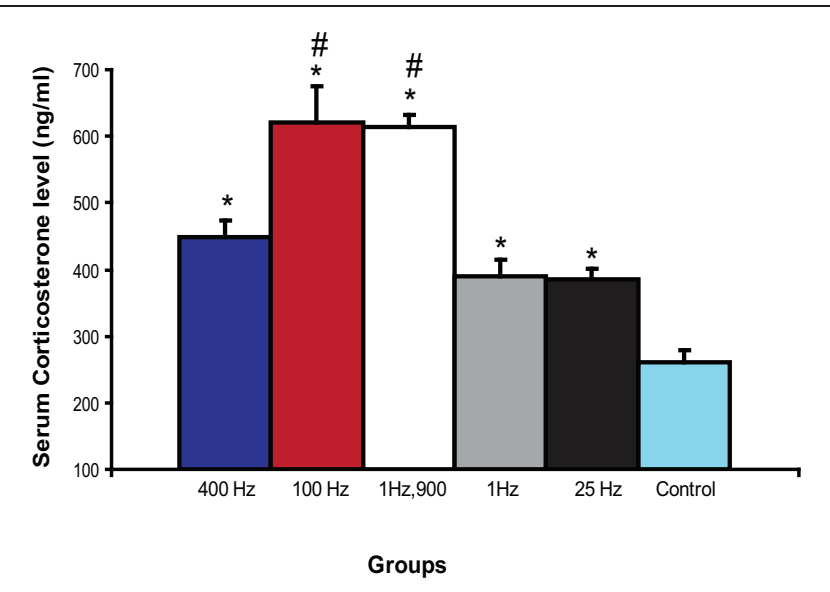

FIGURE 4 | Effect of BLA activation on serum corticosterone.

BLA-stimulated rats showed significantly increased levels of CORT compared with control rats ( $\left.{ }^{*} p<0.01\right)$. In addition, $100 \mathrm{~Hz}$ and $1 \mathrm{~Hz} 900$ groups showed significantly more CORT compared with 1,25 , and $400 \mathrm{~Hz}$ (" $p<0.001)$

\section{DISCUSSION}

Consistent with our previous findings (Vouimba and RichterLevin, 2005; Vouimba et al., 2007), BLA priming revealed a functional dichotomy between effects on LTP in vHC-CA1 synapses and LTP in the PP-DG pathway. Modulation of the strength and frequency of BLA priming stimulation induced changes in LTP in both CA1 and DG. However, while in CA1 BLA priming inhibited LTP, in the DG the effects ranged from impairment to no effect to enhancement of plasticity. Such changes are reminiscent of the effects of various stressors on LTP in the hippocampus.

In accordance with that, modulation of the strength and frequency of BLA priming stimulation resulted in different levels of serum CORT such that CORT levels positively correlated with LTP in DG but not in CA1. Our findings provide further evidence for a differential amygdalar control of plasticity in hippocampal sub-regions. They also provide insight into how various stressors through differential activation of the amygdala may alter memory processes in brain circuitry required for tasks execution.

It is interesting to note that variability in the outcome of stress and of BLA activation is reflected in the DG and not in the CA1. Traditionally, with respect to learning and memory, focus was more on the CA3-CA1 area of the hippocampus, partly because of the impact of the relatively selective ischemic lesions to CA1 (Yamashima et al., 2007) and due to the quality of place cells in these regions (Rolls, 2010). The current results are consistent with recent proposals for a differential role of the DG in aspects of memory, such as pattern separation (Gilbert et al., 2001; Kesner, 2007; Aimone et al., 2011; Schmidt et al., 2012). They add to this view the possibility that DG-associated aspects of memory will be enhanced under more emotional or stressful conditions (Kogan and Richter-Levin, 2008; Segal et al., 2010).

The BLA is known to play a key role in many facets of emotional processing (Fanselow and LeDoux, 1999). Exposure to emotionally arousing experiences enhances BLA neuronal activity. For instance, foot-shock was reported to enhance and synchronize the firing rate of BLA neurons (Pelletier et al., 2005). Moreover, fear-provoking experiences induced lasting potentiation in the BLA (McKernan and Shinnick-Gallagher, 1997; Rogan et al., 1997; Garcia et al., 1998; Vouimba et al., 2004). Correspondingly, learning under stress was found to activate the MAP kinase signal transduction cascade within the BLA (Akirav et al., 2001; Kogan and Richter-Levin, 2008). In humans, neuro-imaging studies and electrophysiological recordings demonstrated strong and reproducible activation of the amygdala in response to fearful stimuli (Whalen et al., 1998; Morris et al., 2002; Oya et al., 2002).

However, stress does not universally enhance BLA neuronal activity. Varying the intensity of an acute stress can lead to different levels of activation of various markers of activity and plasticity in the BLA; e.g., ERKII, CREB (Kogan and Richter-Levin, 2008; Ilin and Richter-Levin, 2009). Some stressors may decrease or have no effect on the BLA. For instance, both acute tail-shocks stress and re-exposure to the stressful context was reported to suppress spontaneous unit activity in the BLA (Shors, 1999). Further, a single exposure or re-exposure to a moderate platform stress inhibited long-lasting LTP in the BLA (Vouimba et al., 2004; Kavushansky et al., 2006). Importantly, clinical studies have found that the amygdala response to fearful faces habituated rapidly over repeated presentations of aversive stimuli (Breiter et al., 1996; Büchel et al., 1998; LaBar et al., 1998).

Stress-induced modulation of BLA activity has been involved in stress modulation of memory processes. Indeed, manipulations which reduce or enhance BLA excitability, respectively, impair or facilitate memory storage (Dickinson-Anson and McGaugh, 1993; Packard et al., 1994; Hatfield and McGaugh, 1999) and synaptic plasticity in the hippocampus (Ikegaya et al., 1996; Akirav and Richter-Levin, 1999; Kim et al., 2001, 2005; Vouimba and Richter-Levin, 2005; Vouimba et al., 2007).

Electrical stimulation of the amygdala is known to produce, in rodents, a complex pattern of behavioral and autonomic changes that resemble fear or aggressive behavior. For example, a brief $200 \mathrm{~Hz}$ stimulation of the amygdala increases the aggressiveness of male Syrian golden hamsters. The effect was sensitive to stimulation amplitude and frequency and lasted for about $30 \mathrm{~min}$ with a peak 10-15 min after stimulation (Potegal et al., 1996). The authors suggested that the persistence of aggression could result from LTP-like changes within the amygdala-related neural circuitry. In humans, amygdala stimulation also elicits feelings of fear, anxiety, and terror (Chapman et al., 1954; Gloor et al., 1981). Early study from Mark and Ervin (1970) showed for example that stimulation of the amygdala with different intensity $(\leq 5 \mathrm{~mA})$ induced either defensive or aggressive behavior. More recently electrical stimulations $(50 \mathrm{~Hz}$, pulse duration $1 \mathrm{~ms}, 5 \mathrm{~s}$ duration; Intensity $0-2.5 \mathrm{~mA}$ ) of the right amygdala, in humans, were reported to induce negative emotions, especially fear and sadness. In contrast, stimulations of the left amygdala were able to induce either pleasant (happiness) or unpleasant (fear, anxiety, sadness) emotions (Lanteaume et al., 2007). It is conceivable that by changing the stimulation characteristics, the authors could have induced a different set of emotions. Although we cannot link our different protocols to a specific behavior, together with the above mentioned studies, our data suggest that the different 
amygdala priming protocol may be related to different emotional state. Thus, stressors-induced different activation patterns at the BLA can be accountable for the wide variety of stress effects on hippocampal LTP and memory processes. Both the nature of the stressor and its temporal attributes could result in differential activation of the amygdala and in a different outcome with regards to changes in both DG and CA1.

Anatomically, there are strong direct and indirect reciprocal connections between the BLA and the hippocampus. However, within the dorsal hippocampal circuitries, the amygdala seems to modulate information processing polysynaptically via its projections to the entorhinal cortex, parasubiculum, and subiculum (Pikkarainen et al., 1999; Pitkänen et al., 2000). Though the different hippocampal sub-regions has been proposed to work together to build a unified memory (Squire and Zola-Morgan, 1991), activation of the BLA, by means of its efferent connections, may favor the selection of some circuits in detriment of others and lead to a qualitatively and quantitatively different memory (Richter-Levin, 2004; Vouimba and Richter-Levin, 2005; Segal et al., 2010).

Another interesting finding was that BLA activation increased CORT levels and that higher levels of CORT were obtained with patterns which also enhanced LTP in DG but not in CA1 (Figure 5). In the control group, CORT level was not correlated with the magnitude of LTP in DG suggesting that amygdala activation is a prerequisite. The amygdala seems to dynamically modulate both CORT and DG-LTP highlighting a sensitivity of both parameters to stressors characteristics, as opposed to the lack of sensitivity and monotonous mode of response of CA1. This result complements our previous findings disclosing dissociation in the mechanism by which the BLA influences hippocampal LTP in DG and CA1. We showed that BLA modulation of LTP in DG, but not in CA1, was dependent on glucocorticoid transmission (GC) in the BLA. Indeed, blocking BLA GC receptors impaired amygdala-dependent enhancement of DG-LTP but had no effect on the impairing effect of BLA on CA1 LTP (Vouimba et al., 2007). Dissociated and bi-directional effects of CORT on DG and CA1 LTP were also reported in studies in which CORT was directly applied in an in vitro hippocampal preparation (Pu et al., 2007).

The complexity of effects of stress on learning and memory is also reflected in the effects of CORT on these processes (de Quervain et al., 2009). CORT, released upon exposure to a stressful event, is known to either enhance or impede memory performance (Sandi et al., 1995; Akirav et al., 2004; de Quervain et al., 2009; Chauveau et al., 2010) as well as activity and plasticity in the hippocampus (McEwen, 1994; Pavlides and McEwen, 1999; Alfarez et al., 2002) and the amygdala (Duvarci and Paré, 2007; Mitra and Sapolsky, 2008). Interestingly, the ability of GC to alter memory processes was found to depend on the cooperation of stress hormones, noradrenaline (NA), and CORT within the BLA (McGaugh, 2000; McGaugh and Roozendaal, 2002). Indeed, while GC and NA agonists into the BLA-facilitated memory formation in a variety of learning paradigms (Roozendaal and McGaugh, 1997) subsequent intra-BLA infusions of their antagonists blocked the memory-enhancing effects (Quirarte et al., 1997; Roozendaal and McGaugh, 1997; Roozendaal et al., 2002).

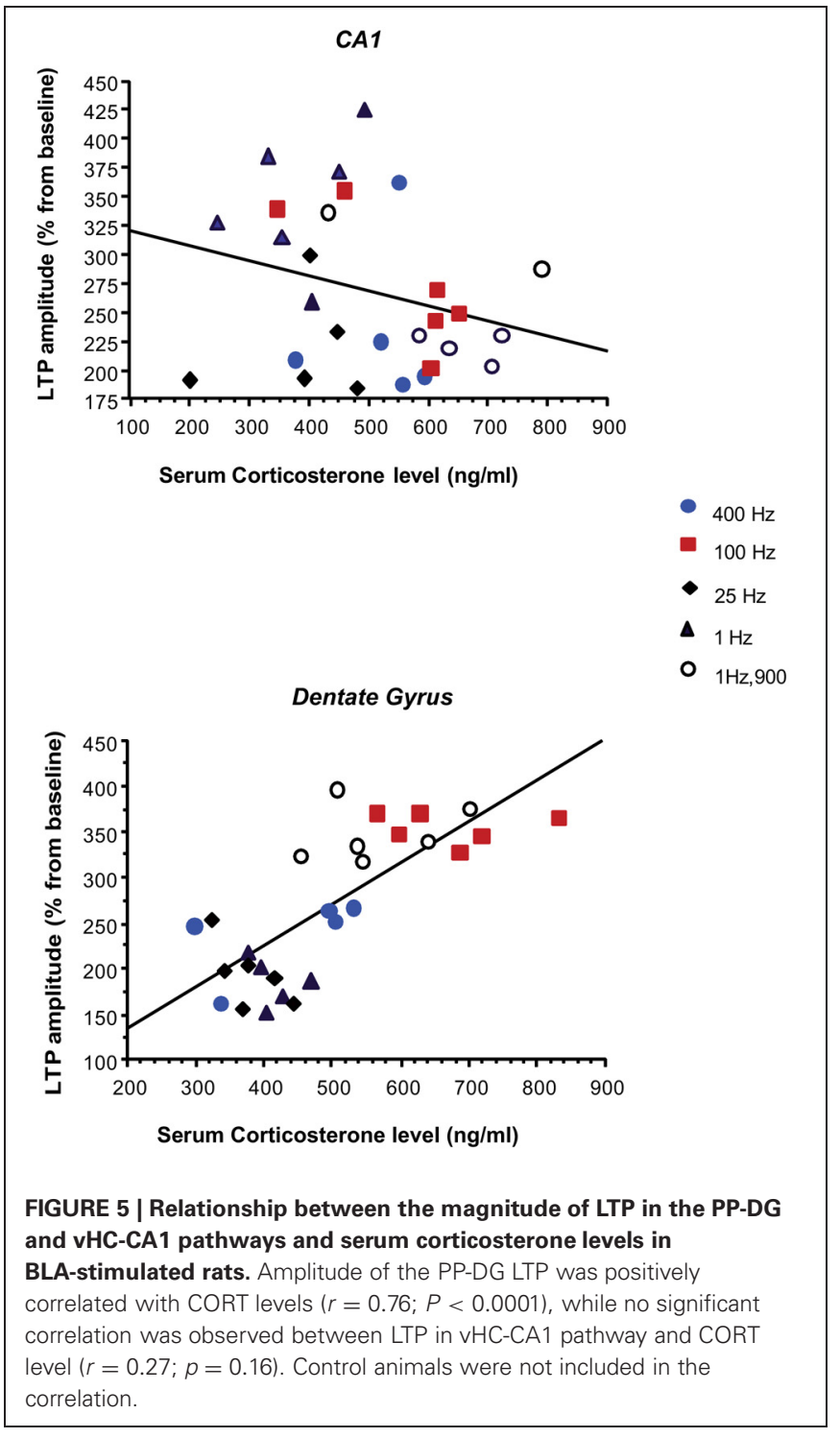

Studies suggest that GC exerts a time-dependent permissive action on the efficacy of the NA system to promote memory (Roozendaal, 2000; Roozendaal et al., 2002). Thus, it is possible that BLA-induced increase in CORT, which alters activity and plasticity in BLA, CA1, and DG, may be mediated in part, via interaction with NA.

However, as was suggested before, the outcome of emotional, stressful, or traumatic experiences would depend on even broader range of interactions (Joëls and Baram, 2009).

In this study, we showed that weak and strong protocols have a differential impact on both DG and CA1 LTP. These protocols have been labeled strong or weak based on the frequency and number of pulses in the protocols. However, the outcome of these protocols, in term of BLA activation is difficult to appraise since we did not measure their direct impact on BLA neuronal activity. Studies from Paré et al. (2003) showed that the amygdala is endowed with a strong inhibitory interface (intercalated cells masses -ITC-) which gates impulse traffic between its input 
and output stations. Stimulation of the BLA was shown to induce, through differential activation of the ITC, both enhancement and inhibition of the main amygdala output. It is therefore not excluded that what we considered a weak pattern may in fact activate whether than inhibit the BLA, depending of their outcome on the ITC. This is congruent with the high level of CORT induced by the $1 \mathrm{~Hz}, 900$ protocol.

It is interesting to think of BLA pattern of activation and the differential levels of circulating CORT as two arms of the emotional and stress response that attempt to synchronize brain activity to best meet the challenge (Kogan and Richter-Levin,
2008; Segal et al., 2010). It is foreseeable to think of abnormal such synchronization under extreme conditions, which would lead to the development of maladaptive behavior.

\section{ACKNOWLEDGMENTS}

This research was supported by an Israel Science Foundation grant number 1403/07 to Gal Richter-Levin, and by the Institute for the Study of Affective Neuroscience, University of Haifa, which was endowed by the Hope for Depression Research Foundation. We thank Emmett Brustel for valuable assistance with corticosterone measures.

\section{REFERENCES}

Abe, K. (2001). Modulation of hippocampal long-term potentiation by the amygdala: a synaptic mechanism linking emotion and memory. Jpn. J. Pharmacol. 86, 18-22.

Aimone, J. B., Deng, W., and Gage, F. H. (2011). Resolving new memories: a critical look at the dentate gyrus, adult neurogenesis, and pattern separation. Neuron 70, 589-596.

Akirav, I., Kozenicky, M., Tal, D., Sandi, C., Venero, C., and Richter-Levin, G. (2004). A facilitative role for corticosterone in the acquisition of a spatial task under moderate stress. Learn. Mem. 11, 188-195.

Akirav, I., and Richter-Levin, G. (1999). Biphasic modulation of hippocampal plasticity by behavioral stress and basolateral amygdala stimulation in the rat. J. Neurosci. 19, 10530-10535.

Akirav, I., Sandi, C., and Richter-Levin, G. (2001). Differential activation of hippocampus and amygdala following spatial learning under stress. Eur. J. Neurosci. 14, 719-725.

Alfarez, D. N., Wiegert, O., Joëls, M., and Krugers, H. J. (2002). Corticosterone and stress reduce synaptic potentiation in mouse hippocampal slices with mild stimulation. Neuroscience 115, 1119-1126.

Baldi, E., and Bucherelli, C. (2005). The inverted "u-shaped" dose-effect relationships in learning and memory: modulation of arousal and consolidation. Nonlinearity Biol. Toxicol. Med. 3, 9-21.

Bliss, T. V., and Collingridge, G. L. (1993). A synaptic model of memory: long-term potentiation in the hippocampus. Nature 361, 31-39.

Bramham, C. R., Southard, T., Ahlers, S. T., and Sarvey, J. M. (1998). Acute cold stress leading to elevated corticosterone neither enhances synaptic efficacy nor impairs LTP in the dentate gyrus of freely moving rats. Brain Res. 789, 245-255.

Breiter, H. C., Etcoff, N. L., Whalen, P. J., Kennedy, W. A., Rauch, S.
L., Buckner, R. L., et al. (1996). Response and habituation of the human amygdala during visual processing of facial expression. Neuron $17,875-887$.

Büchel, C., Morris, J., Dolan, R. J., and Friston, K. J. (1998). Brain systems mediating aversive conditioning: an event-related fMRI study. Neuron 20, 947-957.

Chapman, W. P., Schroeder, H. R., Guyer, G., Brazier, M. A., Fager, C., Poppen, J. L., et al. (1954). Physiological evidence concerning the importance of the amygdaloid nuclear region in the integration of circulating function and emotion in man. Science 120, 949-950.

Chauveau, F., Pierard, C., Coutan, M., Drouet, I., Liscia, P., and Beracochea, D. (2008). Prefrontal cortex or basolateral amygdala lesions blocked the stress-induced inversion of serial memory retrieval pattern in mice. Neurobiol. Learn. Mem. 90, 395-403.

Chauveau, F., Tronche, C., Pierard, C., Liscia, P., Drouet, I., Coutan, M. et al. (2010). Rapid stress-induced corticosterone rise in the hippocampus reverses serial memory retrieval pattern. Hippocampus 20, 196-207.

de Quervain, D. J., Aerni, A., Schelling, G., and Roozendaal, B. (2009). Glucocorticoids and the regulation of memory in health and disease. Front. Neuroendocrinol. 30, 358-370. doi: 10.1016/j.yfrne. 2009.03.002

Diamond, D. M., Bennett, M. C. Fleshner, M., and Rose, G. M. (1992). Inverted-U relationship between the level of peripheral corticosterone and the magnitude of hippocampal primed burst potentiation. Hippocampus 2, 421-430.

Dickinson-Anson, H., and McGaugh, J. L. (1993). Midazolam administered into the amygdala impairs retention of an inhibitory avoidance task. Behav. Neural Biol. 60, 84-87.
Dringenberg, H. C., Oliveira, D., and Habib, D. (2008). Predator (cat hair)-induced enhancement of hippocampal long-term potentiation in rats: involvement of acetylcholine. Learn. Mem. 15, 112-116.

Dunn, J. D., and Orr, S. E. (1984). Differential plasma corticosterone responses to hippocampal stimulation. Exp. Brain Res. 54, 1-6.

Duvarci, S., and Paré, D. (2007) Glucocorticoids enhance the excitability of principal basolateral amygdala neurons. J. Neurosci. 27, 4482-4491.

Fanselow, M. S., and LeDoux, J. E (1999). Why we think plasticity underlying Pavlovian fear conditioning occurs in the basolateral amygdala. Neuron 23, 229-232.

Feldman, S., and Weidenfeld, J. (1998). The excitatory effects of the amygdala on hypothalamopituitary-adrenocortical responses are mediated by hypothalamic norepinephrine, serotonin, and CRF-41. Brain Res. Bull. 45, 389-393.

Foy, M. R., Stanton, M. E., Levine, S., and Thompson, R. F. (1987) Behavioral stress impairs longterm potentiation in rodent hippocampus. Behav. Neural Biol. 48, 138-149.

Garcia, R., Paquereau, J., Vouimba, R. M., and Jaffard, R. (1998). Footshock stress but not contextual fear conditioning induces long-term enhancement of auditory-evoked potentials in the basolateral amygdala of the freely behaving rat. Eur. J. Neurosci. 10, 457-463.

Gerges, N. Z., Stringer, J. L., and Alkadhi, K. A. (2001). Combination of hypothyroidism and stress abolishes early LTP in the CAl but not dentate gyrus of hippocampus of adult rats. Brain Res. 922, 250-260.

Gilbert, P. E., Kesner, R. P., and Lee, I. (2001). Dissociating hippocampal subregions: double dissociation between dentate gyrus and CA1. Hippocampus 11, 626-636.
Gloor, P., Olivier, A., and Quesney, L. F. (1981). "The role of the amygdala in the expression of psychic phenomena in temporal lobe seizures," in The Amygdaloid Complex, ed Y. Ben-Ari (New York, NY: Elsevier/North Holland), 489-507.

Goldstein, L. E., Rasmusson, A. M., Bunney, B. S., and Roth, R. H. (1996). Role of the amygdala in the coordination of behavioral, neuroendocrine, and prefrontal cortical monoamine responses to psychological stress in the rat. J. Neurosci. $16,4787-4798$.

Hatfield, T., and McGaugh, J. L. (1999). Norepinephrine infused into the basolateral amygdala posttraining enhances retention in a spatial water maze task. Neurobiol. Learn. Mem. 71, 232-239.

Ikegaya, Y., Saito, H., and Abe, K. (1996). The basomedial and basolateral amygdaloid nuclei contribute to the induction of longterm potentiation in the dentate gyrus in vivo. Eur. J. Neurosci. 8, 1833-1839.

Ilin, Y., and Richter-Levin, G. (2009) ERK2 and CREB activation in the amygdala when an event is remembered as "Fearful" and not when it is remembered as "Instructive". J. Neurosci. Res. 87, 1823-1831.

Izaki, Y., and Arita, J. (1996). Longterm potentiation in the rat hippocampal CA1 region is inhibited selectively at the acquisition stage of discriminatory avoidance learning. Brain Res. 723, 162-168.

Joëls, M., and Baram, T. Z. (2009). The neuro-symphony of stress. Nat. Rev. Neurosci. 10, 459-466.

Kavushansky, A., Vouimba, R. M., Cohen, H., and Richter-Levin, G. (2006). Activity and plasticity in the CA1, the dentate gyrus, and the amygdala following controllable vs. uncontrollable water stress. Hippocampus 16, 35-42. 
Kesner, R. P. (2007). A behavioral analysis of dentate gyrus function. Prog. Brain Res. 163, 567-576.

Kim, J. J., Koo, J. W., Lee, H. J., and Han, J. S. (2005). Amygdalar inactivation blocks stress-induced impairments in hippocampal long-term potentiation and spatial memory. J. Neurosci. 25, 1532-1539.

Kim, J. J., Lee, H. J., Han, J. S., and Packard, M. G. (2001). Amygdala is critical for stress-induced modulation of hippocampal longterm potentiation and learning. J. Neurosci. 21, 5222-5228.

Kogan, I., and Richter-Levin, G. (2008). Activation pattern of the limbic system following spatial learning under stress. Eur. J. Neurosci. 27, 715-722.

LaBar, K. S., Gatenby, J. C., Gore, J. C., LeDoux, J. E., and Phelps, E. A. (1998). Human amygdala activation during conditioned fear acquisition and extinction: a mixed-trial fMRI study. Neuron 20, 937-945.

Lanteaume, L., Khalfa, S., Régis, J., Marquis, P., Chauvel, P., and Bartolomei, F. (2007). Emotion induction after direct intracerebral stimulations of human amygdala. Cereb. Cortex 17, 1307-1313.

Mark, V. H., and Ervin, F. R. (1970). Violence and the Brain. New York, NY: Harper and Row.

McEwen, B. S. (1994). Corticosteroids and hippocampal plasticity. Ann. N.Y. Acad. Sci. 746, 134-142.

McGaugh, J. L. (2000). Memory a century of consolidation. Science 287, 248-251.

McGaugh, J. L., and Roozendaal, B. (2002). Role of adrenal stress hormones in forming lasting memories in the brain. Curr. Opin. Neurobiol. $12,205-210$.

McKernan, M. G., and ShinnickGallagher, P. (1997). Fear conditioning induces a lasting potentiation of synaptic currents in vitro. Nature 390, 607-611.

Mesches, M. H., Fleshner, M., Heman, K. L., Rose, G. M., and Diamond, D. M. (1999). Exposing rats to a predator blocks primed burst potentiation in the hippocampus in vitro. J. Neurosci. 19, RC18.

Mitra, R., and Sapolsky, R. M. (2008). Acute corticosterone treatment is sufficient to induce anxiety and amygdaloid dendritic hypertrophy. Proc. Natl. Acad. Sci. U.S.A. 105, 5573-5578.

Morris, J. S., deBones, M., and Dolan, R. J. (2002). Human amygdala responses to fearful eyes. Neuroimage 17, 214-222.
Oya, H., Kawasaki, H., Howard, M. A. 3rd., and Adolphs, R. (2002). Electrophysiological responses in the human amygdala discriminate emotion categories of complex visual stimuli. J. Neurosci. 22, 9502-9512.

Packard, M. G., Cahill, L., and McGaugh, J. L. (1994). Amygdala modulation of hippocampaldependent and caudate nucleusdependent memory processes. Proc. Natl. Acad. Sci. U.S.A. 91, 8477-8481.

Paré, D., Royer, S., Smith, Y., and Lang, E. J. (2003). Contextual inhibitory gating of impulse traffic in the intra-amygdaloid network. Ann. N.Y. Acad. Sci. 985, 78-91.

Pavlides, C., and McEwen, B. S. (1999). Effects of mineralocorticoid and glucocorticoid receptors on longterm potentiation in the CA3 hippocampal field. Brain Res. 851, 204-214.

Pavlides, C., Ogawa, S., Kimura A., and McEwen, B. S. (1996). Role of adrenal steroid mineralocorticoid and glucocorticoid receptors in long-term potentiation in the CA1 field of hippocampal slices. Brain Res. 738, 229-235.

Pavlides, C., Watanabe, Y., and McEwen, B. S. (1993). Effects of glucocorticoids on hippocampal long-term potentiation. Hippocampus 3, 183-192.

Paxinos, G., and Watson, C. (1997). The Rat Brain in Stereotaxic Coordinates. 3rd Edn. San Diego, CA: Academic.

Pelletier, J. G., Likhtik, E., Filali, M., and Paré, D. (2005). Lasting increases in basolateral amygdala activity after emotional arousal: implications for facilitated consolidation of emotional memories. Learn. Mem. 12, 96.

Pikkarainen, M., Rönkkö, S., Savander, V., Insausti, R., and Pitkänen, A (1999). Projections from the lateral, basal, and accessory basal nuclei of the amygdala to the hippocampal formation in rat. J. Comp. Neurol. $403,229-260$

Pitkänen, A., Pikkarainen, M. Nurminen, N., and Ylinen, A. (2000). Reciprocal connections between the amygdala and the hippocampal formation, perirhinal cortex, and postrhinal cortex in rat. A review. Ann. N.Y. Acad. Sci. 911, 369-391.

Potegal, M., Hebert, M., DeCoster, M., and Meyerhoff, J. L. (1996). Brief, high-frequency stimulation of the corticomedial amygdala induces a delayed and prolonged increase of aggressiveness in male Syrian golden hamsters. Behav. Neurosci. $110,401-412$.

$\mathrm{Pu}$, Z., Krugers, H. J., and Joëls, M. (2007). Corticosterone time-dependently modulates betaadrenergic effects on long-term potentiation in the hippocampal dentate gyrus. Learn. Mem. 14 359-367.

Quirarte, G. L., Roozendaal, B. and McGaugh, J. L. (1997). Glucocorticoid enhancement of memory storage involves noradrenergic activation in the basolateral amygdala. Proc. Natl. Acad. Sci. U.S.A. 94, 14048-14053.

Richter-Levin, G. (2004). The amygdala, the hippocampus, and emotional modulation of memory. Neuroscientist 10, 31-39.

Rogan, M. T., Stäubli, U. V., and LeDoux, J. E. (1997). Fear conditioning induces associative longterm potentiation in the amygdala. Nature 390, 604-607.

Rolls, E. T. (2010). A computational theory of episodic memory formation in the hippocampus. Behav. Brain Res. 215, 180-196.

Roozendaal, B. (2000). Glucocorticoids and the regulation of memory consolidation. Psychoneuroendocrino$\log y 25,213-238$.

Roozendaal, B., Griffith, Q. K., Buranday, J., De Quervain, D. J., and McGaugh, J. L. (2003). The hippocampus mediates glucocorticoid-induced impairment of spatial memory retrieval: dependence on the basolateral amygdala. Proc. Natl. Acad. Sci. U.S.A. 100, 1328-1333.

Roozendaal, B., Hahn, E. L., Nathan, S. V., de Quervain, D. J., and McGaugh, J. L. (2004). Glucocorticoid effects on memory retrieval require concurrent noradrenergic activity in the hippocampus and basolateral amygdala. J. Neurosci. 24, 8161-8169.

Roozendaal, B., and McGaugh, J. L. (1997). Glucocorticoid receptor agonist and antagonist administration into the basolateral but not central amygdala modulates memory storage. Neurobiol. Learn. Mem. 67, 176-179.

Roozendaal, B., Quirarte, G. L., and McGaugh, J. L. (2002). Glucocorticoids interact with the basolateral amygdala beta-adrenoceptor-cAMP/cAMP/PKA system in influencing memory consolidation. Eur. J. Neurosci. 15 , 553-560.

Sandi, C., Rose, S. P., Mileusnic, R., and Lancashire, C. (1995).
Corticosterone facilitates long-term memory formation via enhanced glycoprotein synthesis. Neuroscience 69, 1087-1093.

Schmidt, B., Marrone, D. F., and Markus, E. J. (2012). Disambiguating the similar: the dentate gyrus and pattern separation. Behav. Brain Res. 226, 56-65.

Segal, M., Richter-Levin, G., and Maggio, N. (2010). Stress-induced dynamic routing of hippocampal connectivity: a hypothesis. Hippocampus 20, 1332-1338.

Shors, T. J. (1999). Acute stress and re-exposure to the stressful context suppress spontaneous unit activity in the basolateral amygdala via NMDA receptor activation. Neuroreport 10, 2811-2815.

Shors, T. J. (2001). Acute stress rapidly and persistently enhances memory formation in the male rat. Neurobiol. Learn. Mem. 75, 10-29.

Squire, L. R., and Zola-Morgan, S. (1991). The medial temporal lobe memory system. Science 20, 1380-1386.

Tsoory, M. M., Vouimba, R. M., Akirav, I., Kavushansky, A. Avital, A., and Richter-Levin, G. (2008). Amygdala modulation of memory-related processes in the hippocampus: potential relevance to PTSD. Prog. Brain Res. 167, 35-51.

Vouimba, R. M., Foy, M. R., Foy, J. G., and Thompson, R. F. (2000). 17beta-estradiol suppresses expression of long-term depression in aged rats. Brain Res. Bull. 53, 783-787.

Vouimba, R. M., Muñoz, C., and Diamond, D. M. (2006). Differential effects of predator stress and the antidepressant tianeptine on physiological plasticity in the hippocampus and basolateral amygdala. Stress 9, 29-40.

Vouimba, R. M., and RichterLevin, G. (2005). Physiological dissociation in hippocampal subregions in response to amygdala stimulation. Cereb. Cortex 15, 1815-1821.

Vouimba, R. M., Yaniv, D., Diamond, D., and Richter-Levin, G. (2004). Effects of inescapable stress on LTP in the amygdala versus the dentate gyrus of freely behaving rats. Eur. J. Neurosci. 19, 1887-1894.

Vouimba, R. M., Yaniv, D., and RichterLevin, G. (2007). Glucocorticoid receptors and beta-adrenoceptors in basolateral amygdala modulate synaptic plasticity in hippocampal dentate gyrus, but not in area CA1. Neuropharmacology 52, 244-252. 
Wang, Y., Rowan, M. J., and Anwyl, R. (1997). Induction of LTD in the dentate gyrus in vitro is NMDA receptor independent, but dependent on $\mathrm{Ca} 2+$ influx via low-voltage-activated $\mathrm{Ca} 2+$ channels and release of $\mathrm{Ca} 2+$ from intracellular stores. J. Neurophysiol. 77, 812-825.

Whalen, P. J., Rauch, S. L., Etcoff, N. L., McInerney, S. C., Lee, M. B., and Jenike, M. A. (1998). Masked presentations of emotional facial expressions modulate amygdala activity without explicit knowledge. J. Neurosci. 18, 411-418.
Xu, L., Holscher, C., Anwyl, R., and Rowan, M. J. (1998). Glucocorticoid receptor and protein/RNA synthesis-dependent mechanisms underlie the control of synaptic plasticity by stress. Proc. Natl. Acad. Sci. U.S.A. 95, 3204-3208.

Yamada, K., McEwen, B. S., and Pavlides, C. (2003). Site and time dependent effects of acute stress on hippocampal long-term potentiation in freely behaving rats. Exp. Brain Res. 152, 52-59.

Yamashima, T., Tonchev, A. B., and Borlongan, C. V. (2007). Differential response to ischemia in adjacent hippocampalsectors: neuronal death in CA1 versus neurogenesis in dentate gyrus. Biotechnol. J. 2, 596-607.

Conflict of Interest Statement: The authors declare that the research was conducted in the absence of any commercial or financial relationships that could be construed as a potential conflict of interest.

Received: 08 October 2012; accepted: 09 April 2013; published online: 03 May 2013.
Citation: Vouimba $R-M$ and RichterLevin G (2013) Different patterns of amygdala priming differentially affect dentate gyrus plasticity and corticosterone, but not CA1 plasticity. Front. Neural Circuits 7:80. doi: 10.3389/fncir. 2013.00080

Copyright (c) 2013 Vouimba and Richter-Levin. This is an open-access article distributed under the terms of the Creative Commons Attribution License, which permits use, distribution and reproduction in other forums, provided the original authors and source are credited and subject to any copyright notices concerning any third-party graphics etc. 